\title{
Beobachtungen der Nova Cygni 1920 im Jahre 1921.
}

Die folgenden Beobachtungen der Helligkeit von Nova Cygni r 920 sind von den Beobachtern der Urania-Sternwarte am Sucher- und Hauptfernrohre des $246 \mathrm{~mm}$-Refraktors angestellt worden. Es handelt sich meistens um Schätzungen. Einige mit einem einfachen Keilphotometer gemachte Beobachtungen sind mit einem Stern bezeichnet.

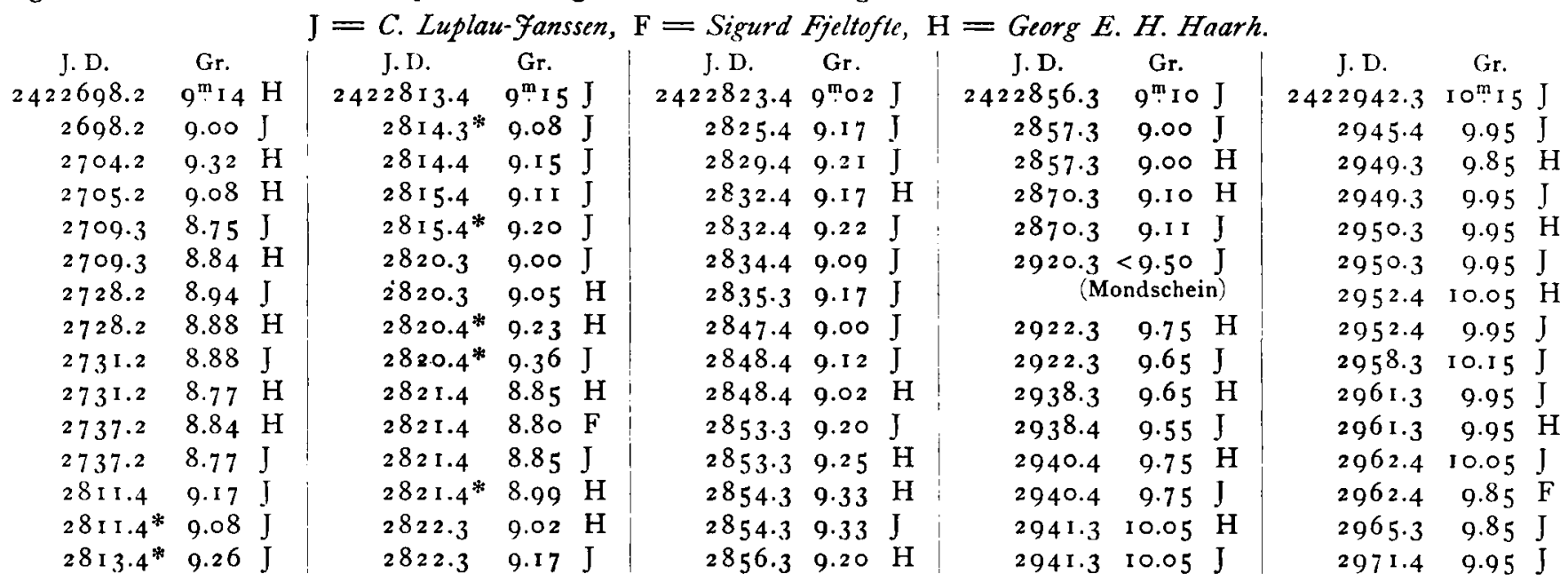

Während des Jahres $\mathrm{I} 92 \mathrm{I}$ beträgt die Lichtabnahme ungefähr ${ }_{\mathrm{I}}{ }^{\mathrm{m}}$.

Urania-Sternwarte, Kopenhagen F., I 92 I Dez. 30.

C. Luplau.Fanssen, G. E. H. Haarh.

\section{Beobachtungen der Nova Aquilae 3 im Jahre 1921.}

Die folgenden Beobachtungen der Nova Aquilae sind im Jahre I92 I auf der Urania-Sternwarte angestellt worden. l)ie meisten Schätzungen sind mit dem Sucher des $246 \mathrm{~mm}$-Refraktors, einige im Hauptrohr desselben Instruments ausgeführt worden. Sowohl die Argelandersche als auch die Harvard-Methode sind verwendet. Einige einzelne mit einem Stern angemerkte Beobachtungen sind mit einem einfachen Keilphotometerapparat ausgefüht worden. Es bedeuten: $\mathrm{J}=$ C. Luplau-Fanssen, $\mathrm{H}=$ Georg $E$. H. Haarh und $\mathrm{F}=$ Sigurd Fjeltofte.

\begin{tabular}{|c|c|c|c|c|c|c|c|c|c|}
\hline $\begin{array}{l}\text { J. D. } \\
228 \text { I } 0.5\end{array}$ & $\begin{array}{l}\text { Gr. } \\
8^{\mathrm{m}} \cdot 95 \mathrm{H}\end{array}$ & $\begin{array}{c}\text { J. D. } \\
2422821.5\end{array}$ & $\begin{array}{l}\text { Gr. } \\
8 \div 95 \mathrm{~F}\end{array}$ & $\begin{array}{c}\text { J. D. } \\
2422835 \cdot 5\end{array}$ & $\begin{array}{c}\text { Gr. } \\
9 \text { mo8 J }\end{array}$ & $\begin{array}{c}\text { J. D. } \\
2422857 \cdot 5\end{array}$ & $\begin{array}{l}\text { Gr. } \\
9 . \circ 8 \quad \text { J }\end{array}$ & $\begin{array}{c}\text { J. D. } \\
2422941.4\end{array}$ & $\begin{array}{c}\mathrm{Gr} \\
9^{\mathrm{m}} \cdot \mathrm{I} 7\end{array}$ \\
\hline 2810.5 & $8.95 \mathrm{I}$ & 2822.5 & $8.95 \mathrm{H}$ & 2847.6 & $9.49 \mathrm{~J}$ & 2875.5 & $8.93 \mathrm{H}$ & 2942.4 & 9.18 \\
\hline $2810.6 *$ & $8.87 \mathrm{H}$ & 2822.5 & $8.95 \mathrm{~J}$ & 2848.5 & $8.98 \mathrm{H}$ & 2875.5 & $8.92 \mathrm{~J}$ & 2942.5 & 9.44 \\
\hline $2810.6 *$ & $8.99 \mathrm{~J}$ & 2823.5 & $9.15 \mathrm{~J}$ & 2848.5 & 9.29 & 2912.5 & $9.56 \mathrm{~J}$ & 2949.5 & 9.17 \\
\hline 28 II. 5 & $8.95 \mathrm{~J}$ & $2823.5 *$ & $9.20 \mathrm{~J}$ & 2848.6 & 9.09 & 2919.5 & $9.49 \mathrm{H}$ & 2949.5 & 9.29 \\
\hline 28 I1. $5 *$ & $9.06 \mathrm{~J}$ & $2825 \cdot 5$ & $9.15 \mathrm{~J}$ & 2853.5 & 9.17 & 2919.5 & $9.49 \mathrm{~J}$ & 2950.5 & 9.29 \\
\hline 28 I 5.5 & $8.95 \mathrm{~J}$ & 2828.5 & 9. Iо H & 2853.5 & 9.29 & 2921.5 & 9.43 & $295^{0.5}$ & 9.29 \\
\hline 28 I $5.6 *$ & $8.87 \mathrm{~J}$ & 2828.5 & $9.05 \mathrm{~J}$ & 2854.5 & $9.09 \mathrm{H}$ & $292 \mathrm{I} .5$ & $9.45 \mathrm{~F}$ & 2959.6 & $9 \cdot 3^{6}$ \\
\hline 2820.5 & $9.05 \mathrm{~J}$ & 2829.5 & $9.60 \mathrm{~J}$ & 2854.5 & 9.1 $4 \mathrm{~J}$ & 2921.5 & $9.50 \mathrm{~J}$ & 2960.5 & $9.4 \mathrm{I}$ \\
\hline $2820.5 *$ & $9.16 \mathrm{~J}$ & 2832.5 & $9.20 \mathrm{H}$ & 2856.5 & 9.23 & 29.38 .4 & 9.37 & 2962.5 & 9.18 \\
\hline 2820.6 & $9.05 \mathrm{H}$ & 2832.5 & $9.15 \mathrm{~J}$ & 2856.6 & 9.29 & & $\left.9.29 H^{2}\right)$ & 2962.5 & 9.17 \\
\hline $2820.6 *$ & $9.02 \mathrm{H}$ & 2834.5 & $9.08 \mathrm{~J}$ & 2857.5 & $\left.9.08 \mathrm{H}^{1}\right)$ & 2938.4 & $\left.9.40 \mathrm{~J}^{1}\right)$ & $2963 \cdot 3$ & 9.53 \\
\hline $282 \mathrm{I} \cdot 5$ & $9.05 \mathrm{H}$ & 2834.6 & $9.09 \mathrm{~J}$ & & 9. I $\left.6 \mathrm{H}^{2}\right)$ & & $\left.9.29 \mathrm{~J}^{2}\right)$ & 2064.3 & 9.66 \\
\hline 2821.5 & $9.00 \mathrm{~J}$ & $28.34 .6^{*}$ & $9.17 \mathrm{~J}$ & 2857.5 & $\left.9.15 \mathrm{~J}^{1}\right)$ & 2941.4 & $9.22 \mathrm{~J}$ & 1) Sucher. ? & Haup \\
\hline
\end{tabular}

Die Nova ist während des Jahres r 92 I immer nebelig gewesen. Ein Kern war aber stets deutlich sichtbar. Gegen Fnde der Beobachtungsperiode schien der Kern indessen etwas schwächer zu werden. Die Nova ist im ganzen schwächer geworden, die Lichtabnahme scheint ungefähr $1 / 2$ Sterngröße zu betragen. Schwankungen sind in unseren Beotachtungen angedeutet. Sie werden später diskutiert werden.

Urania-Sternwarte, Kopenhagen F., I 92 I Dez. 30.

C. Luplau-Fanssen, G. E. H. Haarh.

W Virginis. Um Zusendung unpublizierter Beobachtungen bittet Paul Hiigeler, Berlin SO 33, Schlesische Str. 2 I.

Personalnachrichten. Dr. B. Viaro ist zum Direktor der Sternwarte in Catania als Nachfolger von $A$. Ricio ernannt. - Prof. Dr. F. G. Hagen, S. J., Direktor der Vatikan-Sternwarte, ist zum Socio straniero der R. Accademia dei Lincei gewählt. - Frl. Magistra F.M. Vinter-Hansen wurde unter dem 3 I. Juli I 922 zum Observator der Universitätssternwarte Kopenhagen ernannt. 